\title{
Glück gehabt: Augen-Pfählung blieb ohne Folgen
}

\begin{abstract}
Fremdkörper im Gesichtsbereich sind oft nicht auf den ersten Blick zu erkennen. Im vorgestellten Fall war das Kleinkind, das mit seinem Gesicht auf einen Betonboden fiel, von außen betrachtet eher unauffällig. Erst das CT brachte die Pfählung zum Vorschein.
\end{abstract}

Mit einer Verletzung am rechten Oberlid und einer klaffenden Wunde im Augenwinkel brachten die Eltern ihr zweijähriges Kind in die Universitäts-HNO-Klinik Tübingen (Abb. 1). Das Kind war aus 1,5 m Höhe mit dem Gesicht auf einen Betonboden gestürzt. Leichtes Nasenbluten nach dem Sturz war bereits versiegt. Der Schädel war ansonsten unverletzt und das Kind bei vollem Bewusstsein. Der Allgemeinzustand war leicht reduziert, die Abdomensonographie jedoch unauffällig, berichtet Dr. Natascha Friese in der Zeitschrift „HNO“.

Der rechte Bulbus war intakt, jedoch nach oben rotiert. Eine Rotation mit dem Schielhaken nach unten war nur eingeschränkt möglich, schreibt die Autorin. Bei weiterer Inspektion zeigte sich ein tiefgehender Fremdkörper. Im CT zeigte sich ein spitzer Fremdkörper, der in etwa $1 \mathrm{~cm}$ Tiefe von der rechten Orbita ausgehend, unter Abknickung der Nasenscheidewand, mit seinem lateralen Ende an der Periorbita anlag, diese jedoch nicht verletzt hatte (Abb. 2). Der Abstand zwischen Fremdkörper und Lamina cribrosa betrug $1 \mathrm{~mm}$. Das Corpus alienum, ein $3,8 \mathrm{~cm}$ langes, spitzes Holzstück, wurde endoskopisch entfernt und die Wunde ausgiebig gespült. Sowohl die Periorbita rechts als auch die Dura sowie das Nasennebenhöhlensystem blieben unverletzt. Beide Bulbi standen bei Operationsende wieder gleich hoch. Postoperativ verlief die Behandlung komplikationslos.

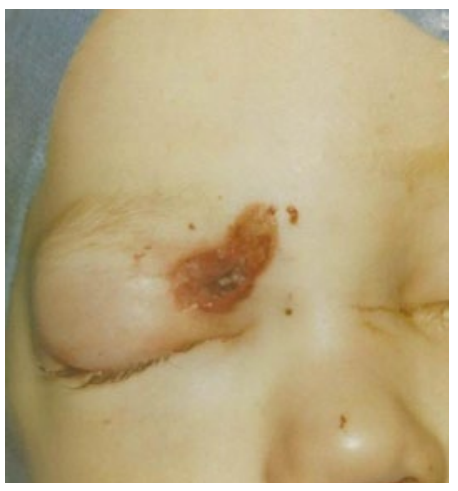

1 Klaffende Wunde im medialen Augenwinkel.

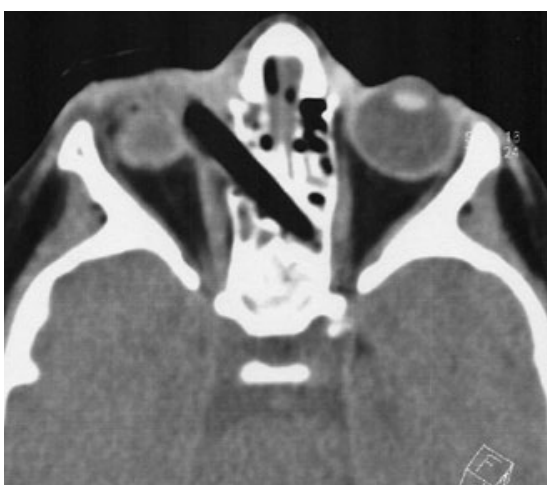

2 CT des Schädels.
Fremdkörper im Gesichtsbereich sind oft nicht auf den ersten Blick zu erkennen. Patienten mit Pfählungsverletzungen im Bereich der Nasennebenhöhlen haben in Anbetracht der Schwere der Verletzung oft wenige, uncharakteristische Symptome. Häufig zeigt sich nur eine scheinbar oberflächliche Verletzung. Besteht der Verdacht auf einen Fremdkörper, ist die CT Mittel der Wahl. $\mathrm{Zu}$ beachten ist, dass sich Holz je nach Wassergehalt wie Luftblasen darstellt - dies kann zu Fehlinterpretationen führen. Die operative Entfernung eines hölzernen Fremdkörpers ist unverzüglich vorzunehmen, da sonst schwerwiegende Komplikationen drohen. Postoperativ muss die Eintrittspforte auf Infektzeichen kontrolliert werden, um Wundinfekte frühzeitig zu erkennen.

(mg)

Friese N, Sturz auf das Gesicht bei 2-jährigem Kind. HNO 2012, 60:827-829
An erster Stelle bei der Suche nach den Ursachen der Mundtrockenheit stehen bildgebende Verfahren wie Ultraschall, Computertomografie und Kernspintomografie. Speichelsteine können meist nur durch die Kombination verschiedener Methoden ausgeschlossen werden. Beschwerden infolge von Schnarchen und Schlaf-Apnoe-Syndrom werden im Schlaflabor abgeklärt. Besteht Verdacht auf ein Sjögren-Syndrom muss die primäre Form mit isolierter Siccasymptomatik von der sekundären unterschieden werden, die zusammen mit anderen Autoimmunerkrankungen auftritt.

Um die Mundtrockenheit zu verbessern, sollte über den ganzen Tag verteilt möglichst viel getrunken werden. Für den
Patienten ist es hilfreich, wenn die Gesamttrinkmenge vorgegeben wird, z. B. zwei Flaschen Mineralwasser täglich. Durch kauen von zuckerfreiem Kaugummi oder das Lutschen von Bonbons lässt sich die Speichelproduktion aktivieren.

\section{Wann künstlicher Speichel?}

Bei starken Beschwerden kann die Speichelproduktion auch medikamentös stimuliert werden (s. Tabelle). Außerdem stehen heute mehrere Produkte für eine Substitution mit künstlichem Speichel zur Verfügung. Sie können beispielsweise $\mathrm{Pa}$ tienten nach einer Radiotherapie in Kombination mit Lidocain-Lösungen Linderung verschaffen. Neben sialagogischen Maßnahmen sollte zudem zu einer beson- ders guten Mundhygiene mit Spülungen geraten werden, weil die verringerte Speichelproduktion das schnelle Fortschreiten einer Strahlenkaries begünstigt.

Da man annimmt, dass der Xerostomie bei Typ-2-Diabetikern eine Mikroangiopathie und Neuropathie zugrunde liegen, ist in diesem Fall möglicherweise eine Behandlung mit Alpha-Liponsäure erfolgreich. Sollte die Mundtrockenheit im Rahmen einer Hypochondrie oder somatoformen Störung auftreten, kann bereits die Aufklärung über die Harmlosigkeit des Symptoms Erleichterung verschaffen.

(Christine Starostzik)

Michel O, Wenn die Spucke wegbleibt. HNO-Nachrichten 2012, 42(5):42-46 